\title{
Source positioning in a large-scale tiny-sensor network of arbitrary topology
}

\author{
Dmitriy Penkin ${ }^{*}$, Gerard Janssen $^{2}$ and Alexander Yarovoy ${ }^{1}$
}

\begin{abstract}
To effectively localise a source node in a dense wireless tiny-sensor network with an arbitrary 2D/3D node distribution, a novel approach suitable to describe the hop progress of source-to-sink path in such a system is proposed. In this approach, the network topology is described as a regular lattice and relates the statistical parameters of the hop count in the source-to-sink propagation path to the fractal properties of a percolating cluster. Based on this approach, a mathematical model is developed to estimate the probability $P(r, t)$ of successful reception of the message by a sink node spaced at distance $r$ from a source in a given time $t$. The accuracy analysis of the positioning method demonstrates its high performance for the very broad spectrum of values of the occupation probability pocc.
\end{abstract}

Keywords: Random network; Lattice model; Percolation theory; Multihop channel; Hop count; Source-to-sink path; Localization algorithm

\section{Introduction}

At present, environmental sensing using electromagnetic waves can be performed using two different approaches. The first approach implies the use of devices that sense an environment remotely (e.g. radars or lidars). Another approach requires a number of sensor nodes, which are distributed in an application area, perform sensing directly inside the phenomenon and wirelessly communicate with each other and with the external reference nodes (sinks). The collected local data are then transmitted through such a wireless sensor network towards an end user in a multihop fashion. The ability to operate and monitor in harsh, complex and inaccessible environments makes the latter approach more promising and ensures a wide range of applications for wireless sensor networks. In particular, there already exist low-end sensor-network-based solutions aiming to detect a sniper location [1], monitor volcano intensity [2], protect forest against fire [3], control water waste in homes [4] and the like [5]. All these actual sensor networks consist of a small number of macro-scale nodes with their positions carefully engineered or pre-determined and capable of coarse-grained sensing. Further development of such

*Correspondence: D.Penkin@tudelft.n

${ }^{1}$ Microwave Sensing, Signals and Systems Group, Delft University of Technology, Mekelweg 4, Delft 2628CD, The Netherlands

Full list of author information is available at the end of the article sensor networks towards a wireless tiny-sensor network (WTSN) with superior fine-grained sensing capabilities would enable a wide spectrum of appealing applications in civil engineering and the health-care sector [6]. Similar to its macro-scale counterpart, each node in a WTSN integrates a sensing, processing and communication unit and a power source [7]. Due to its size restrictions, the tiny node imposes severe constraints on its power component [8]. Since the communication coverage of such a node is thus very limited, the WTSN is foreseen as a swarm of densely deployed nodes within an application area: due to the high node density, the network can still support a long-distance transmission using multihop communication. The simple functionality of tiny nodes implies that the WTSN topology cannot be retrieved (a node has no energy to reconnoitre its surroundings). A large number of these nodes in turn entails that a random scattering over the area to be monitored is the most convenient or perhaps the only option to deploy them. All these features make the WTSN very distinguishable from the current sensor systems. Therefore, novel solutions at the networking level are needed to transfer and extract information gathered by the WTSN.

In particular, an efficient method capable of localising a transmitting node in the WTSN is required. The use of GPS on all nodes is unacceptable in terms of energy resources, node dimensions and its inability to operate

\section{是 Springer}

(c) 2014 Penkin et al:- licensee Springer. This is an Open Access article distributed under the terms of the Creative Commons Attribution License (http://creativecommons.org/licenses/by/2.0), which permits unrestricted use, distribution, and reproduction in any medium, provided the original work is properly cited. 
indoor, so other techniques have to be considered. At present, there are two such major GPS-free techniques for positioning in multihop environments: centralised and distributed localisation. In the first approach, all ranges between all nodes in the network are measured, marked by unique node numbers and are transmitted to a global central unit, which processes and computes the location of each node in the network [9-11]. Evidently, the centralised approach is not a good candidate for the WTSN since it requires a very large communication overhead. Distributed localisation algorithms [12-15] require communication and ranging only with direct neighbouring nodes and are in general more robust and energy efficient than centralised schemes. With distributed localisation, each node in the network is capable of processing and computing to remove a lack of reliance on a single point of possible failure and provide with an optimum load balancing. Both approaches mentioned above require a large amount of energy to perform the prerequisite self-organization stage since the nodes must reconnoitre their surroundings to form a network topology. Due to modest processing capabilities and very limited energy resources of the nodes, the distributed localisation however becomes unaffordable and impracticable for the WTSN. In view of this, we propose a pioneering approach to estimate the location of a source node in the WTSN with arbitrary positions of nodes without consuming extra energy on the self-organization step. This approach employs an analysis of hop progress in such a network and uses time differences of last-arriving signals to its borders to position a source (initially transmitting) node.

Due to the complexity and the unknown locations of sensor nodes, determination of the hop count in the source-to-sink path of the WTSN is intractable with existing solutions, which are based on statistical laws (e.g. the Poisson distribution). Models available in the literature on the probabilistic analysis of the hop count in a large-scale and decentralised system are currently limited to twodimensional (2D) networks. In particular, the probability of establishing a connection of a given length through a planar network with uniformly distributed nodes with a given number of hops has been investigated in [16]. There it was shown that node density does not greatly affect the hop count but has a huge effect on whether a connection can be made at all. In turn, the distribution of Euclidean distances to the $n$th node in a Poisson process is studied in [17]. For homogeneous sensor networks, the connectivity probability in one or two hops is derived and the connectivity in multiple hops is examined with analytical bounds in [18]. In [19], the distribution of the hop distance and its expected value in uniformly distributed networks are analysed by means of numerical simulations. The results indicate that directional nodes might substantially decrease the hop count compared to omnidirectional ones, although the connectivity of a large-scale sensor system will degrade. Hence, as there are merely solutions suitable to determine the hop count in specific 2D WTSN structure, which require heavy computational resources to treat large-scale networks, a different model capable of analysing the source-to-sink multihop path in a 2D/3D WTSN and obtaining its hop count in a lowcost manner is developed here. It is notable that the model capable of analysing the hop progress can also be used to estimate the time required to pass a message through the WTSN and reach a sink node as well as determine other relevant parameters, such as degradation of network throughput and raised source localization cost [20-22].

In the proposed model, the WTSN topology is mapped to a regular lattice, where each lattice site is occupied by a sensor node with certain occupation probability. By relating statistical parameters (such as mean and variance) of the source-to-sink hop count to fractal properties of the percolating cluster in the corresponding lattice, the probability $P(r, t)$ of successful reception of sensed data by a sink node spaced at Euclidean distance $r$ from a source in a given time interval $t$ can in particular be estimated. This probability quantifies the source-to-sink channel in the WTSN and may be regarded as a measure of the network reliability. Based on the hop progress model, the new positioning algorithm that requires no energy on the self-organization step is eventually proposed.

The remainder of the paper is organized as follows. Concepts of percolation theory and the system modelling from the percolation perspective are discussed in Section 2. In Section 3, a technique to determine the hop count of the source-to-sink channel in a WTSN and the probability $P(r, t)$ is developed, whereas simulation and numerical results are provided in Section 4. Finally, the source-positioning method for the WTSN is introduced and discussed in Section 5, whereas the paper concludes in Section 6.

\section{System model}

In the WTSN, the sensed data spreads through the flooding protocol (i.e. a node transmits a signal either when it is triggered by its sensor or when a signal is received from neighbouring nodes [23]) as it is the most suitable way for a network with unknown node topology. To minimize interference overhead, a node is regarded not to broadcast the same message twice if it comes from different transmitting nodes with a delay in time. Each node is assumed to radiate omnidirectionally with a coverage distance $b_{d}$, meanwhile the region covered by the WTSN is represented by a regular lattice structure with a given lattice spacing $a_{d}$ (in the following, the suffix $d$ indicates the dimension). 
In this paper, the $2 \mathrm{D}$ area of interest is discretized in a regular square lattice with $a_{2}=b_{2} / \sqrt{2}$, and thus, the amount of sites to be reached through an one-hop link (i.e. adjacent) is limited by the node degree number $m_{2}=8$ (Figure 1a). In turn, the 3D application space is simplified to a regular cubic lattice with $a_{3}=b_{3} / \sqrt{3}$, and consequently, the number of adjacent sites is limited to $m_{3}=26$ (Figure 1b). Both selected structures are chosen so to be conceptual since any square/cubic lattice with lattice spacing $a_{2}<b_{2} / \sqrt{2}\left(a_{3}<b_{3} / \sqrt{3}\right)$ can be converted to such a corresponding structure using the position-space renormalization group technique and be subsequently resolved [24].

Due to the regularity, the probability that a site of this lattice is occupied by a node is uniform and can be obtained as $p_{\text {occ }}=\rho \cdot\left(a_{d}\right)^{d}$, where $\rho$ is the given node density $\left[\mathrm{m}^{-\mathrm{d}}\right]$, and $\left(a_{d}\right)^{d}$ indicates the $d$-dimensional space occupied by a single site. Two occupied sites are interconnected only if either there exists a one-hop link or both of them share such links with another occupied site. The one-hop link exists solely when the sites are spaced at a distance less than $b_{d}$, while occupied sites separated by a large range (i.e. $\gg b_{d}$ ) can still establish a connection through a multihop channel. The occupied sites form clusters in the lattice. When the occupation probability $p_{\text {occ }}$ is small, there is a sparse population of occupied sites, and clusters composed of small numbers of these sites predominate. However, by increasing $p_{\text {occ }}$, more occupied sites become interconnected and thus become part of the same cluster. Eventually, for $p_{\text {occ }}$ to be large enough, the lattice experiences a critical phase transition: i.e., once $p_{\text {occ }}$ reaches the percolation threshold $p_{c}$, an infinitely large percolating cluster of interconnected sensor sites emerges for the first time [25]. For a finite-sized lattice, this cluster is bounded by the lattice edges, thus also called the spanning cluster [26]. The percolation threshold value $p_{c}$ depends merely on the lattice geometry and the 'lattice' node coverage: the number and organization of sites are interconnected through a one-hop link. Note that due to an exceedingly complex structure of the percolating cluster in higher dimensions, it is cumbersome to determine the percolation threshold analytically except for the 1D and a few 2D lattices [27]. In this respect, the value $p_{c}$ is typically estimated via a numerical experiment.

When $p_{\text {occ }}<p_{c}$, the WTSN is basically fragmented, i.e. composed of many disjoint clusters. Therefore, due to the finite size of a cluster, in which the source node is located, the probability to receive sensed data abruptly goes to zero with increasing source-to-sink separation. In this way, such a sensor network is unreliable and its modelling will not be treated in this paper. Once $p_{\text {occ }}>p_{c}$, the WTSN becomes a dependable system since at any sourceto-sink distance, there always exists a multihop channel between two arbitrary chosen occupied sites when they belong to the percolating cluster. Hence, the probability $P(r, t)$ that a node at Euclidean distance $r$ from a source receives a message in a time interval $t$ can be decomposed as

$$
P(r, t)=p(r, t) \cdot p_{\mathrm{cl}}^{2},
$$

where $p_{\mathrm{cl}}$ is the probability a site belongs to the percolating cluster (the power of two is due to the fact that both source and sink nodes must be part of this cluster), whereas $p(r, t)$ is the probability a signal conveyed through such a cluster is received within the time interval $t$ by the sink node at the distance $r$ from the source. The analysis of $p_{\mathrm{cl}}$ is carried out in Section 4 , while the probability $p(r, t)$ is modelled in the next section.

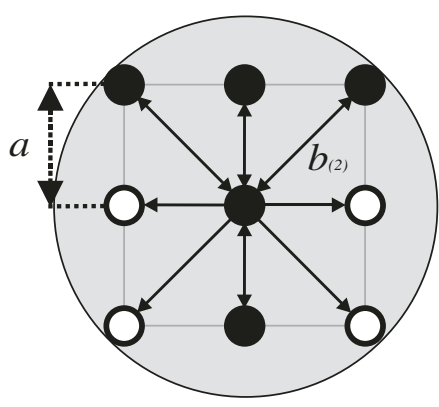

(a)

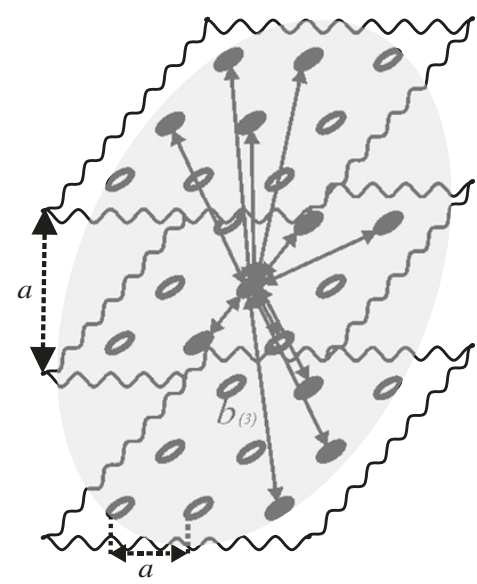

(b)

Figure 1 Node coverage is mapped to a regular lattice at (a) 2D surface and (b) 3D space. Occupied sites are indicated in black, whereas empty ones are drawn in white. 


\section{Signal flooding modelling}

The probability $p(r, t)$ is determined based on the diffusion process defined on the sites of a percolating cluster. In particular, the time variable $t$ is considered discrete. Note that the time unit is determined by the processing delay time in a sensor node $\Delta t$ as it is much larger in general than the propagation time. In other words, at each time unit $\Delta t$, a message is regarded to hop from its current positions to adjacent occupied sites, i.e. the signal propagates in a flooding manner. Hence, in time interval $t$, the sensed signal propagating in a wavefront manner performs $h=\lfloor t / \Delta t\rfloor$ hops and the occupied nodes, which are eventually reached, form an aggregation. For the sake of understanding, such a particular aggregation for $h=20$ in the $2 \mathrm{D}$ lattice of interest with $p_{\text {occ }}=0.5$ is demonstrated in Figure 2.

Since any aggregation is obviously inscribed by a circle (or a sphere for 3D) with its centre coincided with a source site and radius of $h \cdot b$, the probability $p(r, t)$ is a non-zero value only when the number of hops $h$ in time interval $t$ exceeds the minimum hop count $n=\lceil r / b\rceil$ required to cover a distance $r$. To reach a sink node spaced at $r$ in the minimum number of hops $h=n$ with probability 1 , it is required to have no empty sites in a lattice (i.e. $p_{\text {occ }}=1$ ) in order to transmit a signal between sites which are merely located along the source-to-sink direction; thus, the resulting multihop path is a straight line and its fractal degree is equal to 1 . For a lattice with $p_{\text {occ }}<1$, the

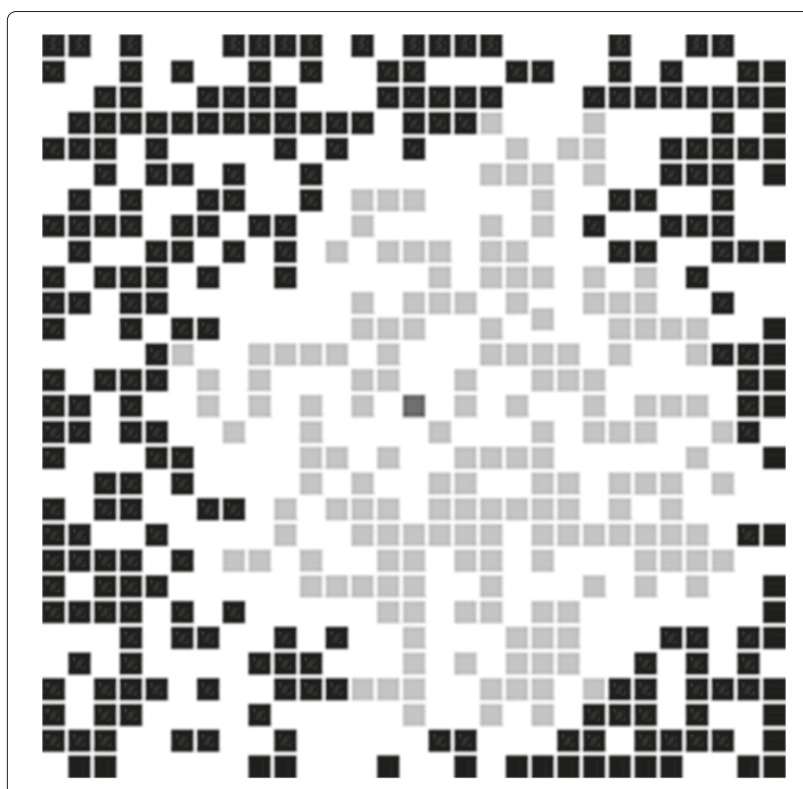

Figure 2 Aggregation of the occupied nodes for $h=20$ in the 2D lattice characterised by $p_{\text {occ }}=\mathbf{0 . 5}$. The blank sites are drawn in white; the occupied sites are shown in black. The sites, which belong to the aggregation, are depicted in light grey, while the origin node is displayed in dark grey. presence of blank sites implies that the fractal degree of the source-to-sink multihop path exceeds 1 , and as a consequence, $h>n$ hops are needed to cover the distance $r$. Evidently, in the worst case scenario, the probability $p(r, t)$ has to be related to the multihop path with the highest fractal degree, which demands the largest number of hops $h$ for reaching the sink at distance $r$. Since such a 'longest' path is composed of the sites situated on the hull of a percolating cluster, statistical parameters of the sourceto-sink path can directly be associated with the fractal properties of this cluster hull. As shown in $[28,29]$ and verified in Section 4, the hop count of a path through a cluster hull is typically distributed according to the Gaussian law. Therefore, the multihop path of interest can be defined in terms of its mean and variance, and the probability $p(r, t)$, which is regarded as the cumulative distribution function of its hop count, is expressed as

$$
p(r, t)=\frac{1}{\sigma \sqrt{2 \pi}} \int_{n}^{h} \exp \left[\frac{(u-\mu)^{2}}{2 \sigma^{2}}\right] d u, h>n,
$$

where $\mu$ and $\sigma$ are the mean and variance corresponding to the hop count in the 'hull' multihop path, respectively. The parameter $h$ is the actual number of hops made in time $t$, while the minimum number of hops needed to cover the source-to-sink distance $r$ is equal to $n$. Hence, the probability $p(r, t)$ increases monotonically from zero $(h<n)$ to $1(h \gg \mu)$, where $\mu$ formally corresponds to the average number of hops to be made to reach the sink node through a 'hull' multihop path.

Since the hull of a percolating cluster does possess fractal properties [29], fractal principles demand that the parameters $\mu$ and $\sigma$, which describe the multihop path composed of sites appertaining to the cluster hull, are expressed via $n$ as follows (this conclusion is also supported through calculations in [30]):

$$
\left\{\begin{array}{l}
\mu=c_{\mu} \cdot n^{d_{\mu}}, \\
\sigma=c_{\sigma} \cdot n^{d_{\sigma}},
\end{array}\right.
$$

where $c$ and $d$ are, respectively, the effective amplitude and the fractal (or Hausdorff) dimension of the Gaussian parameters $\mu$ and $\sigma$. Hence, Equation 2 for $p(r, t)$ can be rewritten using Equation 3 as

$$
\begin{aligned}
p(r, t)= & \frac{1}{c_{\sigma} n^{d_{\sigma} \sqrt{2 \pi}}} \times \\
& \times \int_{n}^{h} \exp \left[\frac{\left(u-c_{\mu} n^{d_{\mu}}\right)^{2}}{2 c_{\sigma}^{2} n^{2 d_{\sigma}}}\right] d u, h>n .
\end{aligned}
$$

As can be seen in Equation 4, the probability $p(r, t)$ can be determined once the effective amplitudes $c_{\mu}, c_{\sigma}$ and the fractal dimensions $d_{\mu}, d_{\sigma}$ are known. Such fractal parameters merely depend on the lattice geometry and 
are estimated by means of numerical experiments (the modelling is simple and shown in Section 4). It is also notable that once these parameters are known, the probability $p(r, t)$ can be assessed for any distance $r$ without extra numerical simulations as the fractal parameters are independent of the source-to-sink separation. This is an essential advantage of the proposed model since; unlike other simulation solutions, it can treat a very large-scale sensor network in a simple way.

\section{Numerical analysis}

The percolation threshold $p_{c}$ and the size of a percolating cluster are numerically explored as both coefficients are of particular interest: the value $p_{c}$ distinguishes percolating from fragmented systems, while its size is directly related to the probability $p_{\mathrm{cl}}$ that an occupied site belongs to the percolating cluster. To determine the fractal parameters related to the cluster hull, the hop count of a multihop path only composed of sites belonging to the hull is investigated as well.

To estimate these parameters, a set of Monte Carlo simulations are carried out. Due to computer limitations, the largest source-to-sink distance of a $2 \mathrm{D}$ lattice being analysed is 2,000 sites, whereas that of a 3D system is bounded by 500 sites. To get accurate results, each set of numerical experiments requires $Q=3,000$ iterations. Per simulation, a signal is only transmitted to the adjacent occupied sites, which are found based on the left-hand maze rule in order to keep its propagation along the cluster hull (by keeping the left hand in contact with one wall of a maze, the player is guaranteed not to get lost and will reach a different exit if there is one). Once there exists a multihop path between the source site and sink spaced at distance $n$, the outcome is assumed to be successful and the hop count $h$ of this path is stored. After applying this procedure $Q$ times, the value $p_{c}$ is determined as the ratio between the number of successful outcomes and the total number of iterations, while the mean value $\mu$ and the standard deviation $\sigma$ are eventually determined through using their common representations.

The percolation threshold of a 2D structure is found to be equal to $p_{c(2)}=0.4073 \pm 0.0007$. This result is in agreement with the data presented in [31]. The percolation threshold of a 3D lattice is significantly less than $p_{c(2)}$ and determined to be $p_{c(3)}=0.0977 \pm 0.0008$. The further estimations are performed under the assumption that the occupation probability $p_{\text {occ }}$ exceeds the percolation threshold to ensure that a percolating cluster arises.

The probability $p_{\mathrm{cl}}$ that a site belongs to the percolating cluster directly corresponds to the ratio of the number of sites in this cluster and the total number of occupied sites. The size of a percolating cluster is determined from numerical simulations on a square and cubic lattice, whereas the amount of occupied sites is consequently expressed as $p_{\text {occ }} \cdot n^{d}$, where $d$ is the lattice dimension. To understand the behaviour of the probability $p_{\mathrm{cl}}$, the size of a percolating cluster is in particular analysed for different values of $p_{\text {occ }}$ and lattice dimensions. For a $2 \mathrm{D}$ and 3D structure, the probability that the starting node belongs to the percolating cluster is found to be reasonably less than 1 in the vicinity of the percolation threshold $p_{c}$ (Figure 3 ). Nonetheless, for both lattices of interest, the value of $p_{\mathrm{cl}}$ rapidly tends to 1 once the occupation probability $p_{\text {occ }}$ increases. In other words, with raising $p_{\text {occ }}$, the level of disjoint clustering in a lattice considerably decreases as more and more occupied sites become part of the percolating cluster. Hence, although $p_{\mathrm{cl}}$ never strictly equals 1 as long as some randomness occurs in a WTSN $\left(p_{\text {occ }}<1\right)$, for practical applications, the probability $p_{\mathrm{cl}}$ can be supposed to be about 1 since a node density would be chosen such that $p_{\text {occ }}$ is reasonably larger than the respective $p_{c}$ for ensuring network connectivity. Note that we however rely on the actual values of $p_{\mathrm{cl}}$ to obtain accurate results here.

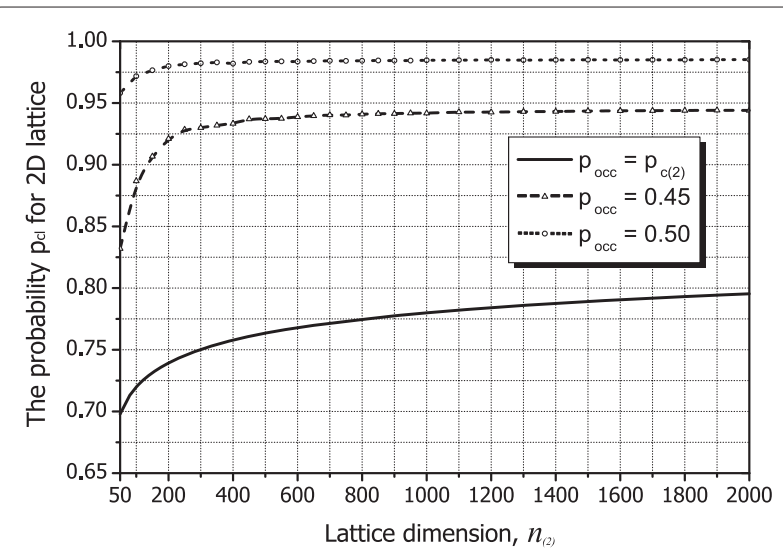

(a)

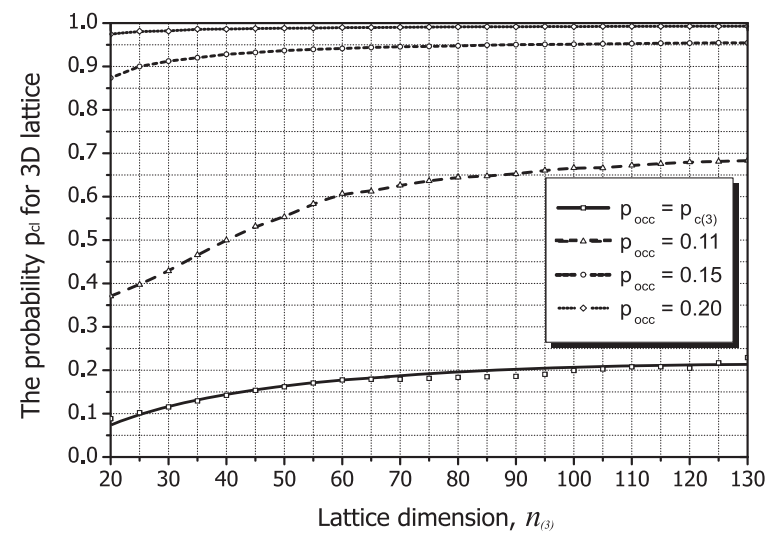

(b)

Figure 3 The probability that a site belongs to the percolating cluster for (a) 2D and (b) 3D lattices of interest. 
For any lattice, the fractal dimensions $d_{\mu}$ and $d_{\sigma}$ are found to reach their maxima when the probability $p_{\text {occ }}$ is near the percolation threshold $p_{c}$ and become minimum when $p_{\text {occ }}$ tends to 1 . The reason for such a behaviour is that by increasing the probability $p_{\text {occ }}$, we decrease the degree of randomness in a network, and consequently, the mean number of hops $\mu$ moves to $n$ as well as the value of $\sigma$ decreases to zero. To demonstrate that the fractal dimension is the prime characteristic which affects the rate of increase of $\mu$ and $\sigma$ with increasing the value $n$, the fractal parameters for both $2 \mathrm{D}$ and $3 \mathrm{D}$ lattices are obtained by fitting the mean and variance of the hop count $h$ calculated for different $n$ to Equation 3. These results are shown in Figure 4 (only for $\mu$ ), whereas the estimated parameters are indicated in Table 1. Also note that such a fractal behaviour is not seen for small hop distances $n$ since the percolation theory requires a great number of entities to become applicable. In particular, according to the numerical experiments, it is suggested to keep $n$ larger than 50 to be able to use this approach.

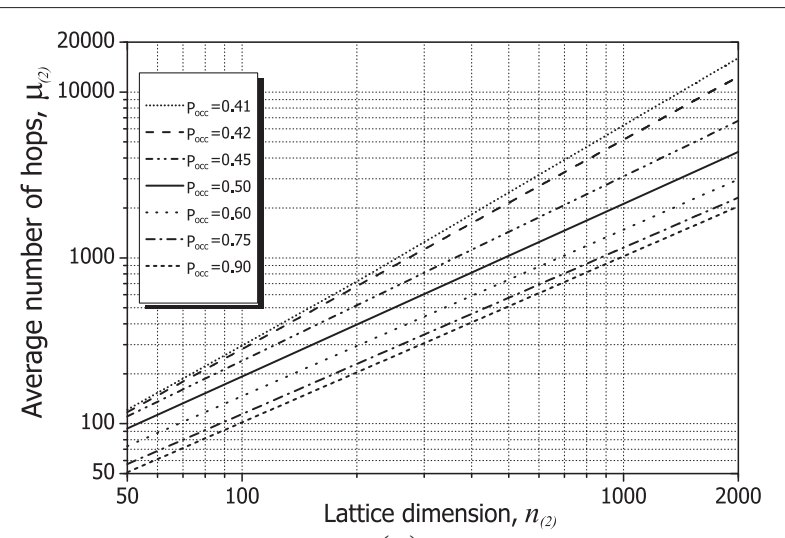

(a)

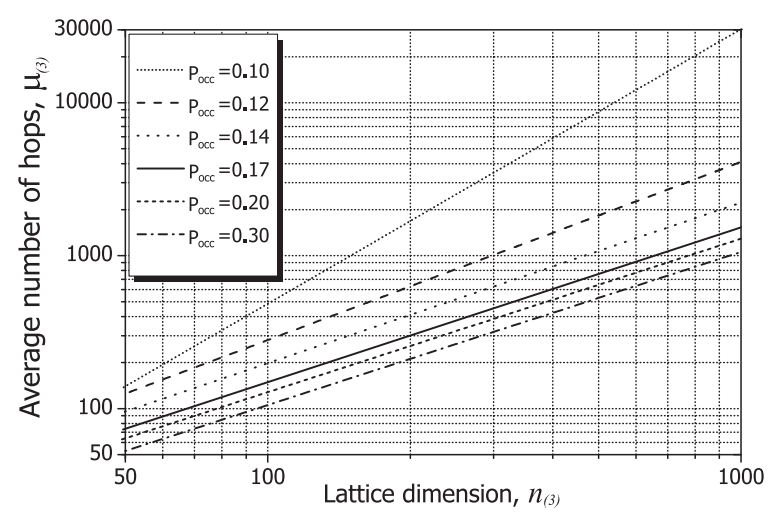

(b)

Figure 4 Dependence of average hop count $\mu$ on lattice dimension for (a) 2D and (b) 3D structures.
Table 1 Fractal characteristics of 2D and 3D lattice structures

\begin{tabular}{ccccccc}
\hline Fractal parameters & \multicolumn{7}{c}{ Values } \\
\hline$p_{\text {occ }(2)}$ & 0.41 & 0.42 & 0.45 & 0.50 & 0.60 & 0.90 \\
$c_{\mu(2)}$ & 0.5736 & 0.8393 & 1.4337 & 1.5947 & 1.4389 & 1.0169 \\
$d_{\mu(2)}$ & 1.3469 & 1.2631 & 1.1116 & 1.0410 & 1.0041 & 1.0007 \\
$c_{\sigma(2)}$ & 0.1252 & 0.2560 & 1.1821 & 1.9558 & 1.6301 & 0.2291 \\
$d_{\sigma(2)}$ & 1.3921 & 1.2470 & 0.8479 & 0.6343 & 0.5178 & 0.5022 \\
$p_{\text {occ }(3)}$ & 0.10 & 0.12 & 0.14 & 0.17 & 0.20 & 0.30 \\
$c_{\mu(3)}$ & 0.1245 & 1.3137 & 1.5666 & 1.4140 & 1.2569 & 1.0556 \\
$d_{\mu(3)}$ & 1.7956 & 1.1650 & 1.0508 & 1.0116 & 1.0041 & 1.0004 \\
$c_{\sigma(3)}$ & 0.0153 & 1.7925 & 2.7176 & 1.9873 & 1.1938 & 0.3684 \\
$d_{\sigma(3)}$ & 2.0603 & 0.8296 & 0.5788 & 0.5038 & 0.5033 & 0.5025 \\
\hline & & & & & & \\
\hline
\end{tabular}

The Kolmogorov-Smirnov criterion is applied to test the suitability of the Gaussian distribution to the random distributions of the hop counts $h_{2}$ and $h_{3}$ for the 2D and 3D systems, respectively. For the sake of curiosity, other major statistical hypotheses are considered as well. In particular, the lognormal distribution fits best both the random distributions of the hop counts. Meanwhile, it has been found that the normal distribution fits well to the calculated random data sets and can fairly describe the arbitrary behaviour of the hop counts $h_{2}$ and $h_{3}$ only if $p_{\text {occ }}$ is more than 0.5 and 0.14 , respectively (Figure 5). Nonetheless, the assumption of normally distributed parameter $p(r, t)$ in Equation 4 is valid, and thus, the model still furnishes proper results for the very broad spectrum of $p_{\text {occ }}$.

To better understand the actual impact of randomness on the WTSN, a 'tortuosity' level of the multihop path along the hull of the percolating cluster is introduced as $\Delta=\mu / n$ and analysed as a function of the probability $p_{\text {occ }}$ and the hop distance $n$ (Figure 6). As can be seen, the larger the distance $n$ and the smaller the probability $p_{\text {occ }}$, the more convoluted is the hop count of the path between a source and sink node. Meanwhile, at large values of $p_{\text {occ }}$, the randomness impact significantly decreases as $\Delta$ is close to 1 and only slightly varies with changing the source-to-sink distance $n$. In other words, when $p_{\text {occ }}$ is in the vicinity of the percolation threshold, the probability $P(r, t)$ becomes negligible since (a) the value of $p_{\mathrm{cl}}$ is small and (b) for a large source-to-sink separation $r$, the probability $p(r, t)$ is also small due to the high tortuosity of the multihop path of interest. Hence, the WTSN with $p_{\text {occ }}$ being about $p_{c}$ seems to be out of interest from a practical perspective. Once $p_{\text {occ }}$ reasonably exceeds the percolation threshold, the probability $P(r, t)$ is mainly determined by the parameter $p(r, t)$ since the value $p_{\mathrm{cl}}$ is close to 1 . For $2 \mathrm{D}$ and $3 \mathrm{D}$ lattices, the probability $P(r, t)$ is calculated and shown in Figure $6 c, d$. As can be seen, the initial value 


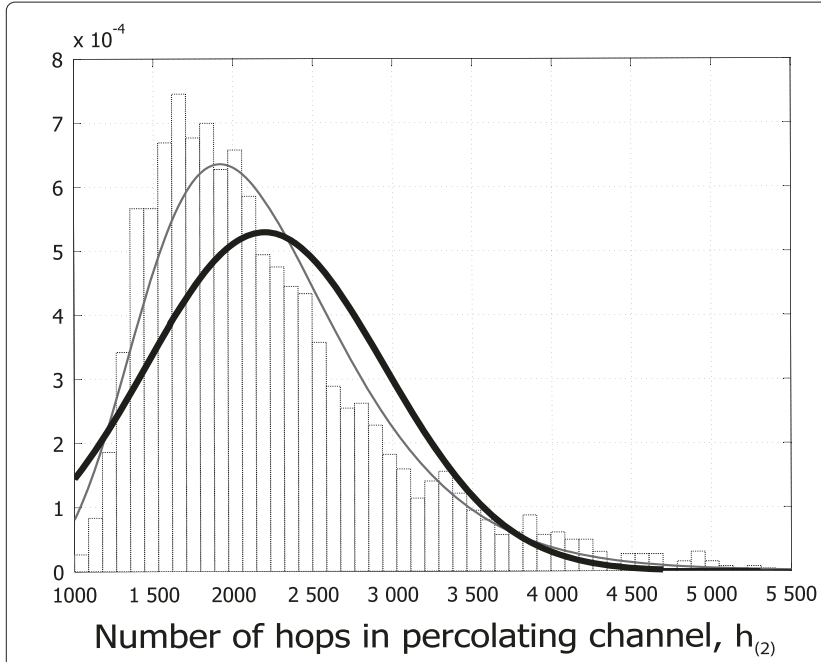

(a)

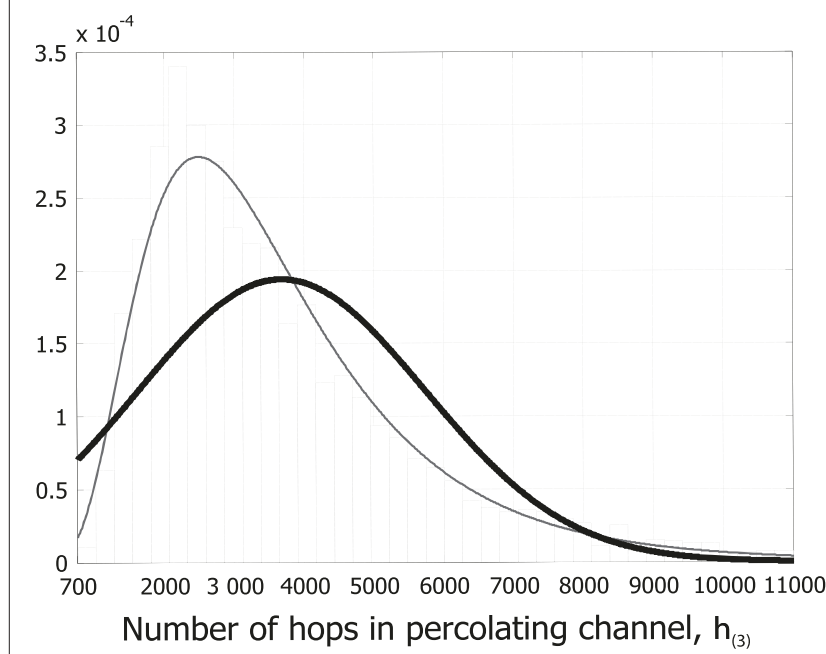

(c)

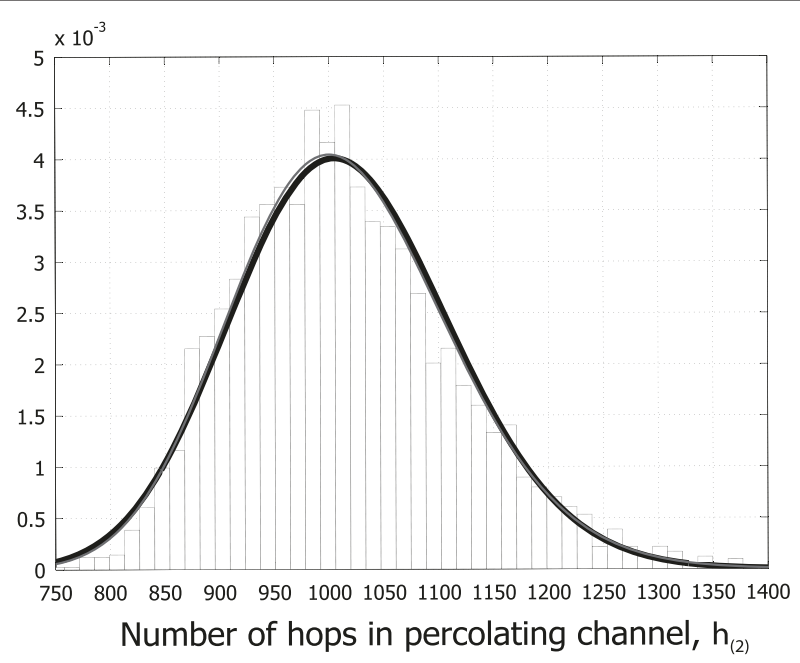

(b)

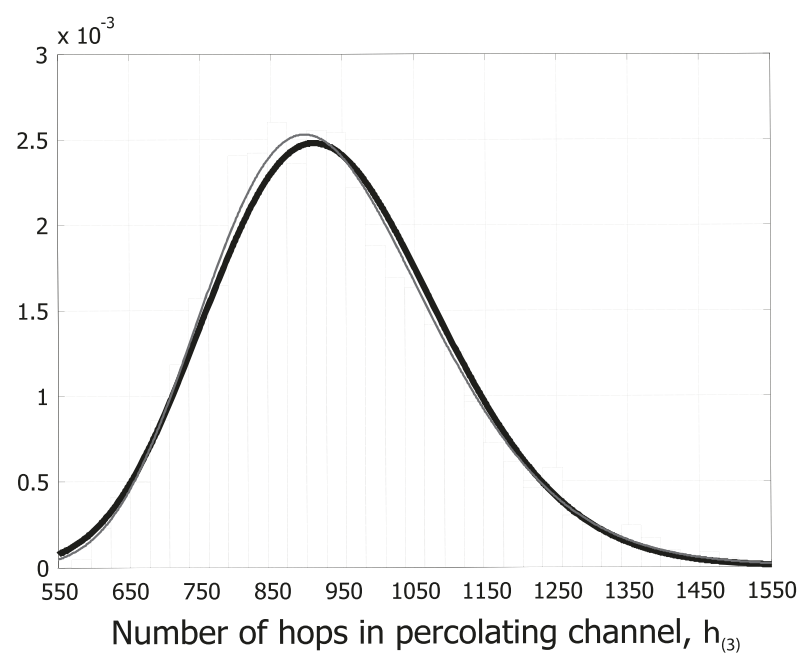

(d)

Figure 5 The random distribution histograms are plotted for the $2 \mathrm{D}$ lattice of dimension $n_{(2)}=500$ with (a) $p_{\text {occ(2) }}=0.42$ and (b) $p_{\text {occ (2) }}=\mathbf{0 . 5 0}$. For the $3 \mathrm{D}$ lattice, the dimension $n_{(3)}=300$ and the occupation probabilities (c) $p_{\text {occ (3) }}=0.10$ and (d) $p_{\text {occ (3) }}=0.14$. A Gaussian distribution approximation is depicted in bold, whereas a line following a lognormal distribution is of standard thickness.

of $p_{\text {occ }}$ considerably affects such a probability: in particular, the value of $P(r, t)$ increases pretty fast by slightly increasing $p_{\text {occ }}$. From this point, it follows that to meet a common criterion of WTSN-based applications, i.e. to receive a signal with a high probability, it is better to add extra sensors and increase the value $p_{\text {occ }}$ rather than expand the time interval $t$. Another fact to be emphasised is that the dimensionality significantly affects the characteristics of a percolating cluster, e.g. $d_{\mu(3)}$ decreases much faster than $d_{\mu(2)}$ with increasing occupational probability due to having an extra degree of freedom in 3D networks. Note also that the Gaussian distribution of the multihop path, which is derived from the results of Monte
Carlo simulations, is depicted in markers in Figure $6 \mathrm{c}, \mathrm{d}$ and corresponds to $p_{\mathrm{occ}(2)}=0.45$ and $p_{\mathrm{occ}(3)}=0.12$, respectively.

Eventually, once the parameters $r$ and $t$ are specified, the lowest density of nodes (i.e. the minimum value of $\left.p_{\text {occ }}\right)$ needed to keep the probability $P(r, t)$ above a given threshold can be determined by using the proposed model. Alternatively, for a given node density and time interval $t$, the distance $r$ in the network can be obtained so that the probability $P(r, t)$ still exceeds some specified level. Since the probability $P(r, t)$ can basically be considered as a good indicator for the performance of a large-scale sensor network, 


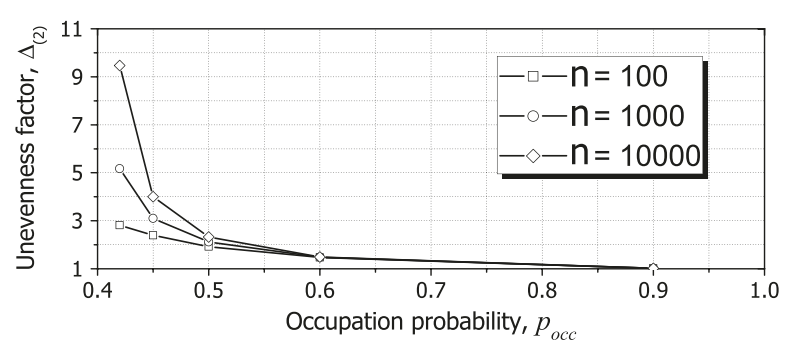

(a)

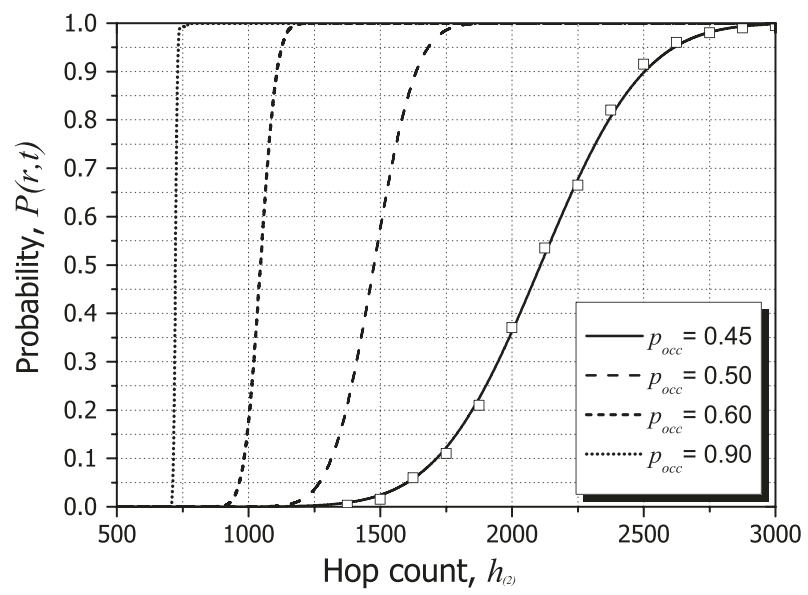

(c)

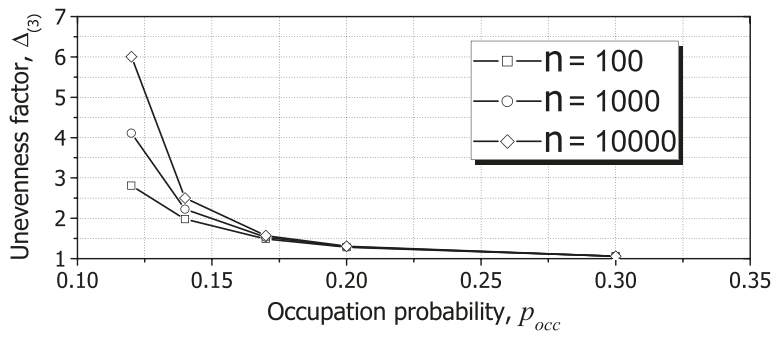

(b)

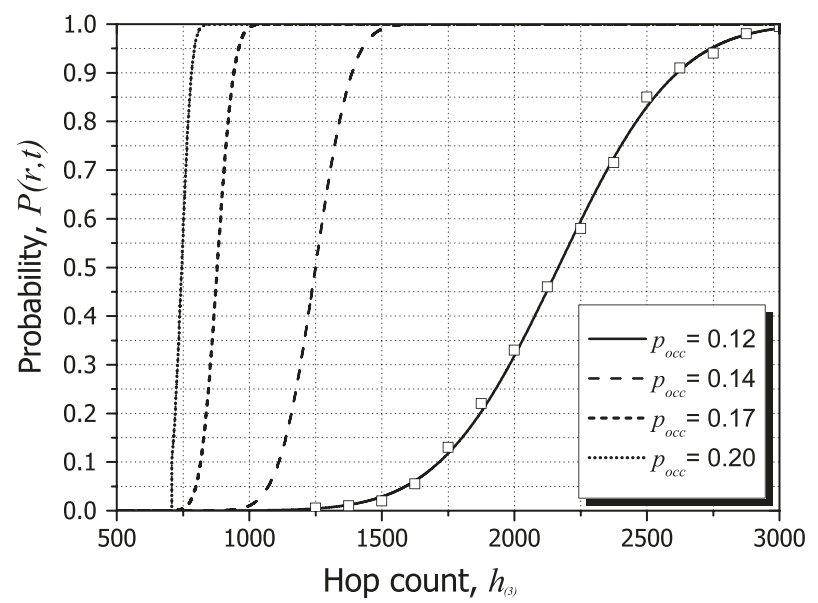

(d)

Figure 6 The tortuosity parameter $\Delta$ and the probability $P(r, t)$ for $2 D(\mathbf{a}, \mathbf{c})$ and $3 D(\mathbf{b}, \mathbf{d})$ lattices. For panels $(\mathbf{c})$ and $(\mathbf{d})$, the source-to-sink separation is $n=700$.

the proposed model is relevant for handling such a system. The source-to-sink path with the maximum number of hops, which can be rigorously defined through the fractal characteristics of the hull of the percolating cluster, is also important from a communication perspective as its modelling helps to avoid inter-message interference in the WTSN (i.e. the source node must send more than one message to complete its report). However, the proposed model is not limited to such predictions on communication properties of a WTSN but also can be used to effectively position a source node in this network.

\section{Source-positioning method}

Once a source node in the WTSN triggers, its position is assumed to be detectable only when this node is located at one of the network borders (i.e. an end user can only get access and treat such 'outward' sensor nodes). If a message originates from one of the 'inward' nodes, there is no direct way to localise this node as it is inaccessible from an end user point of view. Nonetheless, if such an 'inward' source belongs to the percolating cluster, the originating message spreads throughout the
WTSN and is only terminated by the lattice borders. By using the data collected at these borders and applying the developed model of hop progress in the WTSN, the position of the inward source node can reliably be estimated.

The positioning algorithm is, in particular, described for the 3D WTSN. For the sake of simplicity, it is assumed that this area can be represented by a regular lattice of $N_{i} \times N_{j} \times N_{k}$. Once the inward source node with coordinates $(i, j, k)$ belongs to the percolating cluster, a signal originating from it and spreading within this cluster reaches all the lattice borders after all (Figure 7). Hereinafter, let us focus on the determination of parameter $i$ as the values of $j$ and $k$ can be estimated in a similar manner. Evidently, the absolute times of last arriving signals at the left and right borders, denoted as $t_{1}$ and $t_{\mathrm{r}}$, are simply measured (without loss of generality, we may also suppose that $t_{1} \geqslant t_{\mathrm{r}}$ ). As discussed, these time values reflect the product of $\Delta t$ and the hop count of the corresponding peripheral path. In this way, since hop counts $h_{1}$ and $h_{\mathrm{r}}$ are normally distributed parameters, the delay difference time $\Delta t_{i}=t_{1}-t_{\mathrm{r}}$ is also distributed by the Gaussian law, and thus, its mean value $\mu\left(\Delta t_{i}\right)$ 


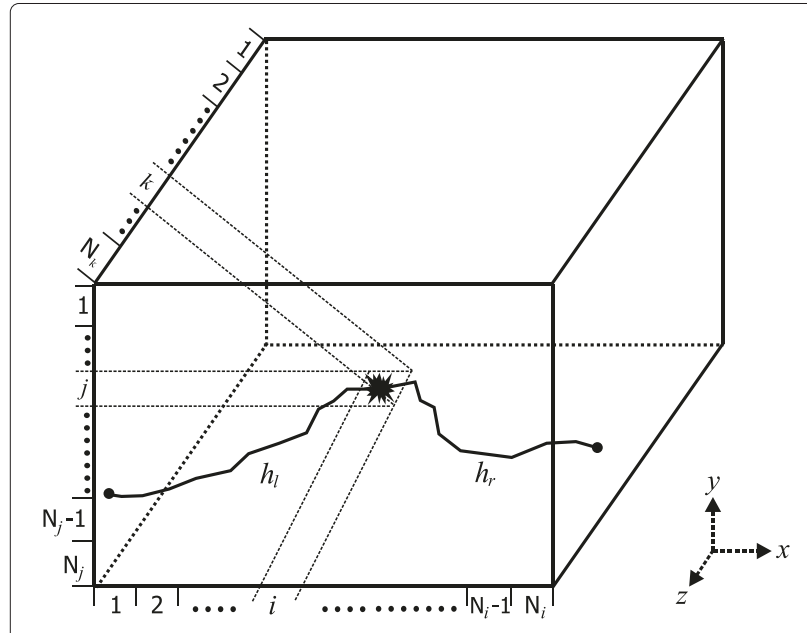

Figure 7 'Hull' multihop paths to the left and right (opposite) borders in a 3D WTSN. The network is simplified by a lattice of dimension $N_{i} \times N_{j} \times N_{k}$, and a source node is situated in $(i, j, k)$ site.

and the standard deviation $\sigma\left(\Delta t_{i}\right)$ can be expressed as follows:

$$
\begin{aligned}
\mu\left(\Delta t_{i}\right) & =\mu\left(t_{1}\right)-\mu\left(t_{\mathrm{r}}\right)=\Delta t\left[\mu\left(h_{1}\right)-\mu\left(h_{\mathrm{r}}\right)\right]= \\
& =\Delta t \cdot c_{\mu(3)}\left[i^{d_{\mu(3)}}-\left(N_{i}-i\right)^{d_{\mu(3)}}\right] ; \\
\sigma\left(\Delta t_{i}\right) & =\sqrt{\sigma\left(t_{1}\right)^{2}+\sigma\left(t_{\mathrm{r}}\right)^{2}}= \\
& =\Delta t \cdot c_{\sigma(3)} \sqrt{i^{2 d_{\sigma(3)}}+\left(N_{i}-i\right)^{2 d_{\sigma(3)}}},
\end{aligned}
$$

where effective amplitudes $c_{\mu(3)}$ and $c_{\sigma(3)}$ and the fractal dimensions $d_{\mu(3)}$ and $d_{\sigma(3)}$ are obtained as described in Section 4. In this context, the coordinate $i$ of the source node can be calculated by measuring the delay times $t_{1}$ and $t_{\mathrm{r}}$ and solving Equation 5 with $\mu\left(\Delta t_{i}\right)=t_{1}-t_{\mathrm{r}}$. Other coordinates $j$ and $k$ can be determined in the similar fashion by assessing the delay times $\Delta t_{j}$ and $\Delta t_{k}$ between the respective lattice boundaries.

From Equation 6, by taking $\partial\left\{\sigma\left(\Delta t_{i}\right)\right\} / \partial i=0$, it follows that the maximum standard deviation in sites for the relative time $\Delta t_{i}$ is given as follows (this derivation is thoroughly discussed in [30]):

$$
\sigma_{\max }\left(\Delta t_{i}\right)=\frac{c_{\sigma(3)} \cdot N_{i}^{d_{\sigma(3)}}}{c_{\mu(3)} \cdot N_{i}^{d_{\mu(3)}}} \cdot N_{i} .
$$

The maximum standard deviation for $\Delta t_{j}$ and $\Delta t_{k}$ is obtained using the same equation but substituting $N_{i}$ with $N_{j}$ and $N_{k}$, respectively. In this regard, the accuracy of the proposed positioning method can be numerically tested by comparing a confidence region of signal origin to the entire lattice area. The confidence region is estimated by using the maximum standard deviation of each of three parameters $\Delta t_{i}, \Delta t_{j}, \Delta t_{k}$ and the normal distribution quantile function (as all these parameters have the Gaussian distribution) and described as

$$
\begin{aligned}
C R_{(3)}= & Z_{p}^{3} \cdot \frac{c_{\sigma(3)} \cdot N_{i}^{d_{\sigma(3)}}}{c_{\mu(3)} \cdot N_{i}^{d_{\mu(3)}}} \cdot \frac{c_{\sigma(3)} \cdot N_{j}^{d_{\sigma(3)}}}{c_{\mu(3)} \cdot N_{j}^{d_{\mu(3)}}} \times \\
& \times \frac{c_{\sigma(3)} \cdot N_{k}^{d_{\sigma(3)}}}{c_{\mu(3)} \cdot N_{k}^{d_{\mu(3)}}} \cdot N_{i} \cdot N_{j} \cdot N_{k},
\end{aligned}
$$

where $Z_{p}$ is the normal distribution quantile for a known confidence level $p$. Since the total number of sites in the lattice is $N_{i} \cdot N_{j} \cdot N_{k}$, the ratio between the confidence region

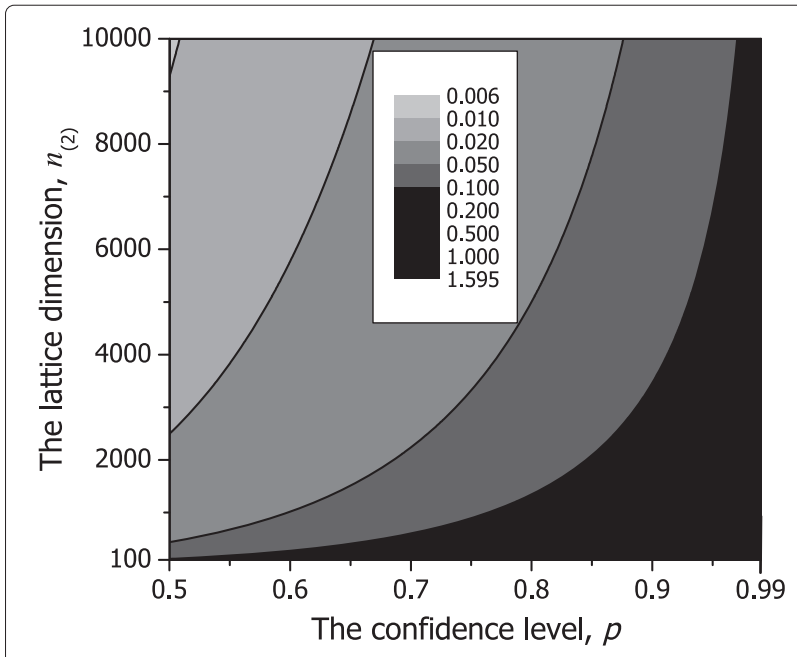

(a)

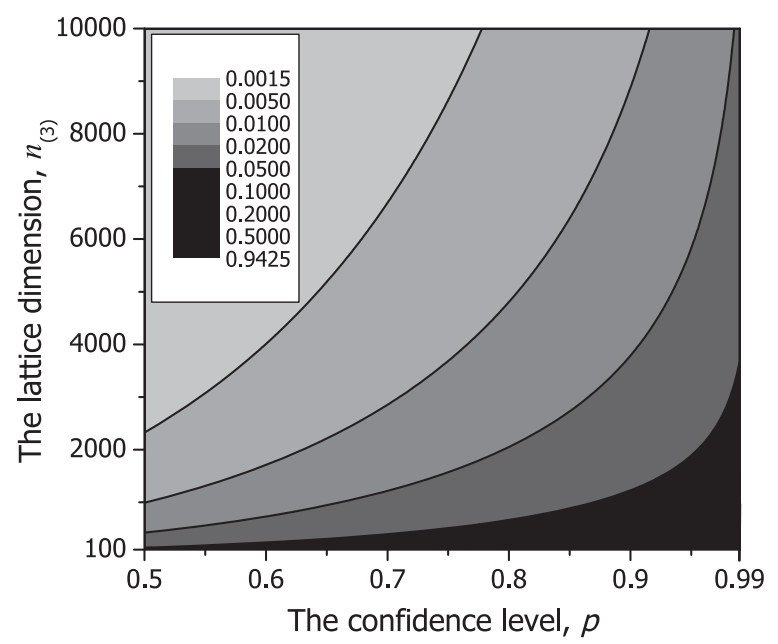

(b)

Figure 8 Accuracy of source localization (the ratio between the confidence area and the entire lattice area). This characteristic is shown as a function of the lattice dimension $n_{(2)}$ and the confidence level $p$ when (a) $p_{\text {occ (2) }}=0.45$ and (b) $p_{\text {occ (2) }}=0.5$ from [32]. 
and the entire lattice area is simplified from Equation 8 as follows:

$$
\Delta_{(3)}=Z_{p}^{3} \cdot \frac{c_{\sigma(3)} \cdot N_{i}^{d_{\sigma(3)}}}{c_{\mu(3)} \cdot N_{i}^{d_{\mu(3)}}} \cdot \frac{c_{\sigma(3)} \cdot N_{j}^{d_{\sigma(3)}}}{c_{\mu(3)} \cdot N_{j}^{d_{\mu(3)}}} \cdot \frac{c_{\sigma(3)} \cdot N_{k}^{d_{\sigma(3)}}}{c_{\mu(3)} \cdot N_{k}^{d_{\mu(3)}}} .
$$

Evidently, the accuracy of the positioning method for the 2D WTSN can be treated in the same way as above. The ratio $\Delta_{(2)}$ is thus reduced from Equation 9 and has the squared $Z_{p}$ and no term containing $N_{k}$ due to the two dimensionality.

The analysis of results suggests that the proposed positioning method technique is limited to the cases when the fractal dimension $d_{\sigma(2,3)}$ is smaller than the value of $d_{\mu(2,3)}$. Otherwise, the algorithm becomes inaccurate and unstable as the value of $\Delta_{(2,3)}$ would diverge with increasing the lattice sizes. This requirement puts a limit on the occupation probability, such as according to Table 1 for the lattices of interest $p_{\text {occ(2) }}>0.42$ and $p_{\text {occ(3) }}>0.11$. Note that due to the fast decrease of $d_{\sigma(2)}$ with increasing occupational probability, the algorithm accuracy noticeably improves once $p_{\text {occ (2) }}$ is moderately larger than the percolation threshold (see Figure 8). For 3D WTSNs, this accuracy refinement is even more substantial due to an extra dimensionality.

\section{Conclusions}

In this paper, we propose a novel method to obtain in a simple manner the worst case number of hops of the source-to-sink path in a very large-scale network with an arbitrary network complexity and the unknown locations of the wirelessly connected nodes. The model assumes that the network topology can be represented by a regular lattice, where each lattice site is occupied by a sensor node with occupation probability $p_{\text {occ }}$. The value $p_{\text {occ }}$ should exceed the percolation threshold $p_{c}$ for the emergence of a percolating cluster. Then, by relating statistical parameters (such as mean and variance) of the hop count of the source-to-sink path to the fractal parameters of the percolating cluster, the probability $P(r, t)$ indicating a successful arrival of the sensed signal to a sink node spaced at distance $r$ from a source within a specified time $t$ has been mathematically expressed. The simple approach to estimate fractal parameters of the percolating cluster based on the left-hand maze rule has been used.

The numerical analysis has been performed for 2D and 3D conceptual lattices to better understand the impact of randomness on a large-scale network. It has been shown that the network with $p_{\text {occ }}$ being close to $p_{c}$ is an unreliable system from an application perspective. Meanwhile, it has also been demonstrated that the occupational probability $p_{\text {occ }}$ greatly affects the level of randomness in the network, i.e. the value $P(r, t)$ increases pretty fast by slightly increasing $p_{\text {occ }}$ beyond $p_{c}$. From this, the practical conclusion can be drawn that it is more effective to increase $P(r, t)$ through adding extra sensors rather than by expanding the time $t$. The raise of $P(r, t)$ with increasing number of sensors is much faster in $3 \mathrm{D}$ network than that in $2 \mathrm{D}$ due to having an extra degree of freedom. The inverse problem, i.e. the determination of the occupational probability needed to maintain the required $P(r, t)$, can also be solved using the developed model. Note that although the model's applicability is currently limited by nodes radiating omnidirectionally, the approach shown in the paper implies that the model can be expanded to nodes with directional coverage once the fractal parameters in the respective lattice structure are estimated.

An effective source-positioning method, which can localise a source node in a large-scale network with arbitrary positions of nodes and without retrieving the network topology, has been proposed based on the developed connectivity model. This localization method, which can be applied in very large scale networks, exploits the fractal nature of the percolating cluster. The accuracy analysis of the method has demonstrated its high performance for the very broad spectrum of values of the occupation probability $p_{\text {occ }}$. As the counterpart approaches are impartially restricted to position in very large-scale networks, this method is the first of its kind to localise a source in these networks and thus is useful for designing such networks in the near future.

\section{Competing interests}

The authors declare that they have no competing interests.

\section{Acknowledgements}

The authors thank the anonymous reviewers for their constructive and helpful comments.

\section{Author details}

${ }^{1}$ Microwave Sensing, Signals and Systems Group, Delft University of Technology, Mekelweg 4, Delft 2628CD, The Netherlands. ${ }^{2}$ Circuits and Systems Group, Delft University of Technology, Mekelweg 4, Delft 2628CD, The Netherlands.

Received: 21 June 2013 Accepted: 9 April 2014

Published: 28 April 2014

\section{References}

1. Á Lédeczi, A Nádas, P Völgyesi, G Balogh, B Kusy, J Sallai, G Pap, S Dóra, K Molnár, M Maróti, Countersniper system for urban warfare. ACM Trans. Sensor Netw. 1(2), 153-177 (2005)

2. G Werner-Allen, K Lorincz, M Ruiz, O Marcillo, J Johnson, J Lees, M Welsh, Deploying a wireless sensor network on an active volcano. IEEE Internet Comput. 10(2), 18-25 (2006)

3. M Hefeeda, M Bagheri, Wireless sensor networks for early detection of forest fires. Paper presented at the, IEEE international conference on mobile ad hoc and sensor systems (Pisa, 8-11 Oct 2007), pp. 1-6

4. Y Kim, T Schmid, ZM Charbiwala, J Friedman, MB Srivastava, NAWMS: nonintrusive autonomous water monitoring system. Paper presented at the 6th, ACM conference on embedded network sensor systems (Raleigh, 4-7 Nov 2008), pp. 309-322

5. Sensor Platform Provider. http://www.libelium.com. Accessed 22 April 2014

6. M llyas, I Mahgoub, Smart Dust: Sensor Network Applications, Architecture and Design (Taylor \& Francis Group, Boca Raton, 2006), p. 352 
7. IF Akyildiz, JM Jornet, Electromagnetic wireless nanosensor networks. Nano Commun. Networks. 1(1), 3-19 (2010)

8. SRoundy, D Steingart, L Frechette, PK Wright, JM Rabaey, ed. by H Karl, A Wolisz, and A Willig, Power sources for wireless sensor networks, in Lecture Notes in Computer Science: Wireless Sensor Networks (Springer, Berlin, 2004), pp. 1-17

9. Y Shang, W Ruml, Y Zhang, MP Fromherz, Localization from mere connectivity. Paper presented at 4 th international symposium on nobile ad hoc networking and computing (Annapolis, 1-3 June 2003), pp. 201-212

10. L Doherty, KSJ Pister, L Ghaoui, Convex position estimation in wireless sensor networks. Paper presented at the 20th conference of the, IEEE Computer and Communications Societies, vol. 3 (Anchorage, 22-26 April 2001), pp. 1655-1663

11. G Giorgetti, SK Gupta, G Manes, Wireless localization using self-organizing maps. Paper presented at the 6th international conference on information processing in sensor networks (Cambridge, 25-27 April 2007), pp. 293-302

12. THe, C Huang, BM Blum, JA Stankovic, T Abdelzaher, Range-free localization schemes for large scale sensor networks. Paper presented at the 9th international conference on mobile computing and networking (San Diego, 14-19 Sept 2003), pp. 81-95

13. K Langendoen, N Reijers, Distributed localization in wireless sensor networks: a quantitative comparison. Comput. Network. 43(4), 499-518 (2003)

14. CSJ Rabaey, K Langendoen, Robust positioning algorithms for distributed ad hoc wireless sensor networks. Paper presented at the USENIX technical annual conference (Monterey, 10-15 June 2002), pp. 317-327

15. G Mao, B Fidan, BDO Anderson, Wireless sensor network localization techniques. Comput. Network. 51(10), 2529-2553 (2007)

16. SAG Chandler, Calculation of number of relay hops required in randomly located radio network. Electron. Lett. 25(24), 1669-1671 (1989)

17. M Haenggi, On distances in uniformly random networks. IEEE Trans. Inform. Theor. 51(10), 3584-3586 (2005)

18. C Bettstetter, J Eberspacher, Hop distances in homogeneous ad hoc networks. Paper presented at the IEEE vehicular technology conference, vol. 4 (Jeju, 22-25 April 2003), pp. 2286-2290

19. R Vilzmann, C Bettstetter, D Medina, C Hartmann, Hop distances and flooding in wireless multihop networks with randomized beamforming. Paper presented at the 8th international symposium on modeling, analysis and simulation of wireless and mobile systems (Montreal, 10-13 Oct 2005), pp. 20-27

20. AE Gamal, J Mammen, B Prabhakar, D Shah, Throughput-delay trade-off in wireless networks. Paper presented at the 23rd conference of the IEEE Computer and Communications Societies, vol. 1 (Hong Kong, 7-11 March 2004), pp. 464-475

21. J Li, C Blake, DSJ De Couto, HI Lee, R Morris, Capacity of ad hoc wireless networks. Paper presented at the 7th annual international conference on mobile computing and networking (Rome, 16-21 July 2001), pp. 61-69

22. Q Chen, Analysis and Application of Hop Count in Multi-hop Wireless Ad Hoc Networks. PhD Thesis, University of New South Wales, 2009

23. ZJ Haas, JY Halpern, L Li, Gossip-based ad hoc routing. IEEE/ACM Trans. Netw. 14(3), 479-491 (2006)

24. Y Yuge, A renormalisation group approach for two-dimensional site percolating system. Journal of Physics A: Mathematical and General. 11(4), L83-L85 (1978)

25. D Stauffer, A Aharony, Introduction to Percolation Theory, 2nd edition. (Taylor \& Francis Group, Philadelphia, 1994), p. 192

26. D Avraham, S Havlin, Diffusion and Reactions in Fractals and Disordered Systems (Cambridge University Press, Cambridge, 2005), p. 332

27. R Ewing, A Hunt, Percolation theory: topology and structure, in Percolation Theory for Flow in Porous Media, 3rd edn. (Springer, Berlin, 2014), pp. 1-35

28. S Vural, E Ekici, Analysis of hop-distance relationship in spatially random sensor networks. Paper presented at the 6th ACM international symposium on mobile ad hoc networking and computing (Chicago, 25-28 May 2005), pp. 320-331

29. J Feder, Fractals (Plenum Press, New York, 1988), p. 283

30. D Penkin, A Yarovoy, G Janssen, A study on communication aspects of two-dimensional large-scale wireless sensor networks using percolation principles. Paper presented at the 17th IEEE symposium on communications and vehicular technology in the Benelux (Enschede, 24-25 Nov 2010), pp. 1-6

31. C-C Shen, Z Huang, C Jaikaeo, Directional broadcast for mobile ad hoc networks with percolation theory. IEEE Trans. Mobile Comput. 5(4), 317-332 (2006)

32. D Penkin, G Janssen, A Yarovoy, Source node location estimation in large-scale wireless sensor networks. Paper presented at the 42nd IEEE European microwave conference (Amsterdam, 29 Oct-1 Nov 2012), pp. 333-336

doi:10.1186/1687-6180-2014-57

Cite this article as: Penkin et al: Source positioning in a large-scale tiny-sensor network of arbitrary topology. EURASIP Journal on Advances in Signal Processing 2014 2014:57.

\section{Submit your manuscript to a SpringerOpen ${ }^{\circ}$ journal and benefit from:}

- Convenient online submission

- Rigorous peer review

- Immediate publication on acceptance

- Open access: articles freely available online

- High visibility within the field

- Retaining the copyright to your article

Submit your next manuscript at $\boldsymbol{\nabla}$ springeropen.com 